\title{
MODELAGEM E SIMULAÇÃO DE UM SISTEMA MASSA-MOLA COM 2 GRAUS DE LIBERDADE
}

\author{
C. Cancian; P. Domingues
}

\section{INTRODUÇÃO}

O presente texto tem como objetivo apresentar a resolução e uma discussão dos aspectos teóricos envolvidos no Exercício de Modelagem $e$ Simulação Computacional 2 (EMSC 2) de 2015, que fez parte da disciplina PME3200 - Mecânica II.

No exercício, foi realizada a modelagem matemática do seguinte sistema: uma esfera de massa $\mathbf{m}$, aproximada como um ponto material $\mathbf{P}$, que poderia se movimentar sem atrito por um plano horizontal; essa massa estaria ligada aos pontos A e B, em paredes fixas e que distam $\boldsymbol{a}$ do ponto de equilíbrio $\mathbf{O}$, por duas molas ideais e idênticas de comprimento natural $\boldsymbol{l}$ e constante $\boldsymbol{k}$.



Figura 1: Sistema em estudo

A ideia era estudar a dinâmica da massa, ao deslocá-la arbitrariamente do equilíbrio, como ilustrado na figura ao lado. Isto é, buscar entender como os diversos parâmetros envolvidos influenciavam nas equações de movimento do sistema; estudar a sua estabilidade e os pontos de equilíbrio; e, finalmente, realizar uma série de simulações para comparar com os resultados analíticos.

Assim, reproduziremos todo o estudo realizado, bem como adicionaremos uma maior discussão tanto dos conceitos físicos e matemáticos envolvidos na modelagem, quanto da interpretação dos resultados das simulações e a relação com outros sistemas análogos de interesse. 


\section{EQUACIONAMENTO DO SISTEMA}

A modelagem do sistema foi realizada utilizando-se as equações de movimento de Lagrange. Para um sistema com N coordenadas generalizadas as equações de Lagrange podem ser dadas por:

$$
\frac{d}{d t}\left(\frac{\partial L}{\partial \dot{q}_{k}}\right)-\frac{\partial L}{\partial q_{k}}=Q_{k}, \text { para } k=1,2, \ldots, N
$$

Em que $q_{k}$ é a k-ésima coordenada generalizada, $Q_{k}$ a força generalizada corresponde e $\mathrm{L}=\mathrm{T}-\mathrm{V}$ a lagrangeana do sistema, sendo $\mathrm{T}$ a energia cinética e $\mathrm{V}$ a energia potencial.

No sistema do EMSC é possível escolher como coordenadas generalizadas as coordenadas usuais $(x, y)$, que descrevem completamente o seu movimento. Com essas coordenadas, tem-se que:

i. Energia Cinética - sendo a massa $(\mathrm{m})$, um ponto material $\mathrm{P}(x, y)$ tem sua energia cinética dada por:

$$
T(\dot{x}, \dot{y})=\frac{1}{2} m\left(\dot{x}^{2}+\dot{y}^{2}\right), \text { sendo } \dot{x}=\frac{d x}{d t} \text { e } \dot{y}=\frac{d y}{d t}
$$

ii. Energia Potencial - a energia potencial será devida exclusivamente às molas, sendo, portanto, da forma: $V=\frac{1}{2} K\left(\Delta L_{A}^{2}+\Delta L_{B}^{2}\right)$, em que $\Delta L_{i}$ é a variação do comprimento de cada uma das molas (A e B). Mas, vale que:

$$
\begin{aligned}
\Delta \vec{L}_{A} & =(P-A)-\vec{j}=x \vec{i}+[(y-a)-l] \vec{j} \\
\Delta \vec{L}_{B} & =(P-B)-\vec{j}=x \vec{i}+[(y+a)-l] \vec{j}
\end{aligned}
$$

E assim, finalmente, chega-se em:

$$
V(x, y)=\frac{1}{2} K\left[\left(\sqrt{x^{2}+(y-a)^{2}}-l\right)^{2}+\left(\sqrt{x^{2}+(y+a)^{2}}-l\right)^{2}\right]
$$

Tendo as energias cinética e potencial, estamos em condição de escrever a lagrangeana do sistema, que será dada por:

$$
L=T(\dot{x}, \dot{y})-V(x, y)=\frac{1}{2} m\left(\dot{x}^{2}+\dot{y}^{2}\right)-\frac{1}{2} K\left[\left(\sqrt{x^{2}+(y-a)^{2}}-l\right)^{2}+\left(\sqrt{x^{2}+(y+a)^{2}}-l\right)^{2}\right]
$$


Como não há forças não-conservativas atuando, tem-se todas as informações necessárias para aplicar as equações de Lagrange às coordenadas $(x, y)$, de modo a obter as equações de movimento do sistema:

$$
\left\{\begin{array} { l } 
{ \frac { d } { d t } ( \frac { \partial L } { \partial \dot { x } } ) - \frac { \partial L } { \partial x } = 0 } \\
{ \frac { d } { d t } ( \frac { \partial L } { \partial \dot { y } } ) - \frac { \partial L } { \partial y } = 0 }
\end{array} \Rightarrow \left\{\begin{array}{l}
m \ddot{x}+K\left[2 x-l\left(\frac{x}{\sqrt{x^{2}+(y-a)^{2}}}+\frac{x}{\sqrt{x^{2}+(y+a)^{2}}}\right)\right]=0 \\
m \ddot{y}+K\left[2 y-l\left(\frac{y-a}{\sqrt{x^{2}+(y-a)^{2}}}+\frac{y+a}{\sqrt{x^{2}+(y+a)^{2}}}\right)\right]=0
\end{array}\right.\right.
$$

De cara, já é possível perceber que o sistema de equações diferenciais obtidos é extremamente não-linear, dado que os termos originários do potencial foram responsáveis pelo aparecimento de raízes do quadrado das coordenadas envolvidas em cada uma das equações.

A não-linearidade dessas equações, por sua vez, permite que se imagine um comportamento caótico das suas soluções e, além disso, que os fenômenos associados a esse comportamento caótico sejam influenciados pela configuração das molas. Isto é, se as molas estão inicialmente comprimidas, relaxadas ou alongadas. Afinal, isso influencia no potencial gerado e, consequentemente, na linearidade das equações de movimento.

Nesse sentido, justifica-se a introdução, desde já, do parâmetro $\alpha$ (real e positivo), definido por: $\alpha=l / a$

Ou seja, esse parâmetro é a razão entre o comprimento natural $(l)$ da mola pela distância do ponto $\mathrm{O}$ às paredes $(a)$, de modo que, para três intervalos de valores de $\alpha$ é possível associar um significado físico importante:

$$
\left\{\begin{array}{l}
0<\alpha<1 \Rightarrow \text { molas alongadas } \\
\alpha=1 \Rightarrow \text { molas relaxadas } \\
\alpha>1 \Rightarrow \text { molas comprimidas }
\end{array}\right.
$$

\section{ANÁLISE DO EQUILÍBRIO}

Com essas definições, é possível iniciar a investigação da dinâmica do sistema. De maneira geral, é uma boa ideia iniciar a análise através dos pontos de equilíbrio, uma vez que, fisicamente, esses pontos são aqueles em que, ao se liberar o sistema com velocidade inicial nula, esse sistema permanecerá em sua configuração inicial para todo instante de tempo. Ou seja, matematicamente, pontos de equilíbrio representam as soluções constante das equações de movimento. 
Nesse sentido, podemos estabelecer uma condição para encontrar os configurações de equilíbrio. Um ponto $\mathrm{P}_{0}=\left(\mathrm{q}_{1}{ }^{0}, \ldots, \mathrm{q}_{\mathrm{N}}{ }^{0}\right)$ é dito ponto de equilíbrio de um sistema com $\mathrm{N}$ coordenadas generalizadas se satisfaz $\mathrm{o}$ conjunto de $\mathrm{N}$ equações dadas por:

$$
\left(\frac{\partial V}{\partial q_{k}}\right)_{P_{0}}=0 \quad, \forall k=1,2, \ldots, N
$$

Veja que, de fato, isso nos garante soluções constantes para um sistema liberado com velocidade inicial nula. Suponha um sistema conservativo genérico, de modo que a lagrangeana (na maioria dos casos clássicos) é dada por:

$$
L=\frac{1}{2} m\left(\dot{q}_{1}^{2}+\dot{q}_{2}^{2}+\ldots+\dot{q}_{N}^{2}\right)-V\left(q_{1}, q_{2}, \ldots, q_{N}\right)
$$

A equação de movimento para a k-ésima coordenada será então:

$$
m \ddot{q}_{k}+\left(\frac{\partial V}{\partial q_{k}}\right)=0
$$

Que é, simplesmente, a segunda lei de Newton. Pelo nosso critério de equilíbrio, se no ponto $\mathrm{P}_{0}$ a derivada parcial se anula para todo $\mathrm{k}$ chegamos a conclusão que a aceleração em cada coordenada também é zero e como, por hipótese, o sistema é liberado do repouso, ele assim permanecerá para sempre. Isto é, a solução da equação de movimento de fato é constante e igual a $\mathrm{P}_{0}$.

É importante notar que nesse caso do sistema conservativo, o critério de equilíbrio se traduz em encontrar os pontos em que a força conservativa se anula, uma vez que (para $q_{1}, \ldots, q_{N}$ coordenadas cartesianas usuais):

$$
\vec{F}=-\nabla V, \text { com } \nabla=\left(\frac{\partial}{\partial q_{1}} \vec{q}_{1}, \frac{\partial}{\partial q_{2}} \vec{q}_{2}, \ldots, \frac{\partial}{\partial q_{N}} \vec{q}_{N}\right)
$$

Ou seja, se em um ponto a derivada do potencial se anula para todo $\mathrm{k}$, isso significa que o gradiente é nulo e, assim, a força atuante no sistema é nula nesse ponto em questão (que é justamente a noção fundamental de equilíbrio mecânico). De qualquer forma, o critério apresentado continua válido em situações um pouco mais gerais e a melhor maneira de interpretá-lo é pensando que os pontos de equilíbrio devem respeitar a condição de serem 
extremantes da função potencial, o que pode não significar necessariamente força nula, como será possível ver mais adiante.

Vamos, então, achar os pontos de equilíbrio do sistema proposto no EMSC usando esse critério. Isto é, precisamos resolver o sistema de equações dado por:



Por inspeção, não é difícil de verificar que o ponto $\mathrm{P}_{0}=(0,0)$ é solução do sistema. Isso é um indicativo de que nossas considerações sobre equilíbrio são razoáveis, afinal esse ponto é justamente o ponto $\mathrm{O}$, recebendo, por isso, o nome de equilíbrio trivial.

Além desse ponto, para $x=0$, é possível ver, usando a equação (2), que os pontos $(0, l)$ e $(0,-l)$ também são soluções do sistema. Veja que esses dois pontos representam a massa nas posições A e B, respectivamente. No entanto, é estranho imaginar que esses fossem pontos de equilíbrio, já que, como as duas molas são idênticas e estão sob solicitações opostas (uma completamente comprimida e a outra completamente esticada) deveria se esperar uma força resultante máxima e tendendo a expulsar a massa desses pontos. Na realidade, o que ocorre é uma espécie de equilíbrio dinâmico, cuja análise foge do nosso interesse nesse texto.

Por último, para $y=0$, realizando um certo trabalho algébrico, fica claro que é preciso ter $x= \pm a \sqrt{\alpha^{2}-1}$ para que o sistema acima seja válido (lembrando que definimos o parâmetro $\alpha$ como $\alpha=a / l$ ), o que nos dá mais 2 pontos de equilíbrio.

Desses cinco pontos encontrados, os dois últimos são os mais interessantes, pois existe uma restrição embutida na sua existência: $\alpha \geq 1$, uma vez que estamos nos limitando a valores reais de $x$ e $y$. Ou seja, conforme variamos o parâmetro $\alpha$ (o que, lembre-se, significa alterar o estado de tensão inicial das molas) obteremos diferentes pontos de equilíbrio no nosso sistema.

Assim, se $\alpha<1$ (as molas estão alongadas), teremos apenas a origem e os pontos simétricos ao eixo $x$ como sendo de equilíbrio. Para $\alpha=1$ (molas relaxadas) as soluções $\left(0, \pm a \sqrt{\alpha^{2}-1}\right)$ se reduzem a um único ponto que coincide com a origem, de modo que o equilíbrio é similar ao caso anterior. Evidentemente, para $\alpha>1$ (molas comprimidas) essas soluções se tornam dois pontos simétricos em relação a origem e que estão sob o eixo $y$. Conforme $\alpha$ 
cresce, ou seja, quanto mais alongadas ficam as molas, esses pontos de equilíbrio se afastam um do outro.

Um comportamento como esse, em que determinadas condições de equilíbrio variam com um parâmetro, recebe o nome de bifurcação, mas também é possível encontrar o termo quebra (espontânea) de simetria. Esses nomes são bastante adequados, afinal, analisando nosso caso, o que acontece é que temos um único ponto de equilíbrio no eixo $y$ até o valor crítico de $\alpha=1$, quando as posições de equilíbrio se dividem em duas e se afastam (bifurcam), como é possível ver no chamado diagrama de bifurcação abaixo:



Diagrama de bifurcação do parâmetro $\alpha$

\section{ANÁLISE DE ESTABILIDADE}

Sendo conhecidos os pontos de equilíbrio o caminho mais natural a seguir é tentar classificá-los quanto a sua estabilidade, afinal, ainda mais importante do que conhecer os pontos em que o sistema pode se equilibrar é conseguir decidir para quais desse pontos o equilíbrio é "mais natural".

Mais precisamente, ao estudarmos estabilidade estamos interessados em saber qual é o efeito de pequenas perturbações no sistema, que retiram-no de suas posições de equilíbrio. A nossa classificação dependerá, então, da forma com que o sistema evolui a partir dessa pequena perturbação: se o sistema tender a voltar ao ponto de equilíbrio, teremos o chamado equilíbrio estável; caso contrário, isto é, se o sistema se afasta arbitrariamente desse ponto, teremos um equilíbrio instável.

O interessante dessa ideia de "pequenas perturbações" é que ela imediatamente se relaciona com a ideia de linearização. Isso porque, se estamos considerando quantias pequenas, os termos quadráticos ou de ordem mais elevada acabam se tornando pequenos o suficiente para serem desprezados.

Assim, o que podemos fazer é juntar as ideias de análise de estabilidade e linearização: vamos linearizar as equações de movimento e estudar sua 
estabilidade em torno dos pontos de equilíbrio encontrados; a partir disso, poderemos extrapolar nossas conclusões para o sistema original.

Uma possibilidade de se proceder para linearizar as equações é através das próprias equações de movimento de Lagrange, isto é, tentar garantir que, ao utilizarmos essas equações, tenhamos como resultado equações lineares. Para isso, é importante olhar a forma geral das equações de Lagrange para um sistema conservativo qualquer de $\mathrm{N}$ dimensões (com a lagrangeana explicitada):

$$
\frac{d}{d t}\left(\frac{\partial}{\partial \dot{q}_{k}}(T-V)\right)-\frac{\partial}{\partial q_{k}}(T-V)=0
$$

O ponto fundamental da linearização está no efeito, em $\mathrm{T}$ e $\mathrm{V}$, das operações de derivação realizadas ao se aplicar essas equações. Em primeiro lugar, deriva-se $\mathrm{T}$ e $\mathrm{V}$ com relação às velocidades generalizadas. Ora, o potencial conservativo $\mathrm{V}$, em geral, é assumido como sendo função apenas das posições, de modo que se anula:

$$
V=V\left(q_{1}, \ldots, q_{N}\right) \Rightarrow \frac{\partial V}{\partial \dot{q}_{k}}=0, \forall k
$$

Por outro lado, para vermos o que ocorre com a energia cinética $\mathrm{T}$, é preciso fazer algumas considerações extras para reescrevê-la de forma conveniente. Para um sistema com n partículas, por exemplo, T é assumida como sendo da forma:

$$
T=\frac{1}{2} \sum_{i} m_{i} \vec{v}_{i} \cdot \vec{v}_{i}
$$

Em que $\vec{v}_{i}$ é a velocidade da i-ésima partícula. Mas, sendo a velocidade a derivada temporal do vetor posição $\vec{r}_{i}$ que, por sua vez, é função das $\mathrm{k}$ coordenadas generalizadas e, eventualmente, do tempo, ficamos com:

$$
\begin{aligned}
& \vec{r}_{i}=\vec{r}_{i}\left(q_{1}, \ldots, q_{N}, t\right) \Rightarrow \vec{v}_{i}=\frac{d \vec{r}_{i}}{d t}=\sum \frac{\partial \vec{r}_{i}}{\partial q_{k}} \dot{q}_{k}+\frac{\partial \vec{r}_{i}}{\partial t} \\
& T=\frac{1}{2} \sum_{i} m_{i}\left(\sum_{k} \frac{\partial \vec{r}_{i}}{\partial q_{k}} \dot{q}_{k}+\frac{\partial \vec{r}_{i}}{\partial t}\right)\left(\sum_{l} \frac{\partial \vec{r}_{i}}{\partial q_{l}} \dot{q}_{l}+\frac{\partial \vec{r}_{i}}{\partial t}\right)
\end{aligned}
$$


Acima usamos a regra da cadeia para derivar $\vec{r}_{i}$ e substituímos o resultado obtido na expressão anterior para a energia cinética. Abrindo a equação obtida, chegamos em:

$$
T=\frac{1}{2} \sum_{i} m_{i} \frac{\partial \vec{r}_{i}}{\partial t} \frac{\partial \vec{r}_{i}}{\partial t}+\sum_{i} \sum_{k} m_{i} \frac{\partial \vec{r}_{i}}{\partial t} \frac{\partial \vec{r}_{i}}{\partial q_{k}} \dot{q}_{k}+\frac{1}{2} \sum_{i} \sum_{k, l} m_{i} \frac{\partial \vec{r}_{i}}{\partial q_{k}} \frac{\partial \vec{r}_{i}}{\partial q_{l}} \dot{q}_{k} \dot{q}_{l}
$$

Mas, na maioria dos casos $\vec{r}_{i}$ não depende explicitamente do tempo, de modo que a energia cinética se reduz a:

$$
T=\frac{1}{2} \sum_{i} \sum_{k, l} m_{i} \frac{\partial \vec{r}_{i}}{\partial q_{k}} \frac{\partial \vec{r}_{i}}{\partial q_{l}} \dot{q}_{k} \dot{q}_{l}=\frac{1}{2} \sum_{k, l} m_{k l} \dot{q}_{k} \dot{q}_{l}, \text { com } m_{k l}=\sum_{i} m_{i} \frac{\partial \vec{r}_{i}}{\partial q_{k}} \frac{\partial \vec{r}_{i}}{\partial q_{l}}
$$

Finalmente, a expressão de $m_{k l}$ sugere que podemos reescrever essa expressão em uma notação matricial, uma vez que é possível interpretar k e l como índices das linhas e colunas, respectivamente, de uma matriz $\mathrm{NxN}$ (afinal, temos $\mathrm{N}$ coordenadas generalizadas), chamada matriz de massa (M).

Definindo, então, $\dot{\boldsymbol{q}}$ (sem subscrito) como sendo o vetor de velocidades generalizadas, obtemos a seguinte expressão matricial para a energia cinética de um sistema de partículas:

$$
\dot{q}^{t}=\left[\begin{array}{lll}
\dot{q}_{1} & \ldots & \dot{q}_{N}
\end{array}\right] \Rightarrow T=\frac{1}{2} \dot{q}^{t} M \dot{q}
$$

Agora estamos em condições de avaliar o efeito de derivarmos $\mathrm{T}$ em relação à velocidade generalizada nas equações de Lagrange. Lembrando que estamos interessados em linearizar em torno do ponto do equilíbrio, é possível perceber que essa informação sobre a posição influencia eventualmente apenas no valor da matriz de massa. Por isso, chamando simplesmente de $m_{k l}$ os elementos $m_{k l}^{P_{0}}$ da matriz de massa avaliados no ponto de equilíbrio $\mathrm{P}_{0}$, teremos:

$$
\frac{\partial T}{\partial \dot{q}_{k}}=\frac{\partial}{\partial \dot{q}_{k}}\left(\frac{1}{2} \dot{q}^{t} M \dot{q}\right)=\sum_{l} m_{k l}^{P_{0}} \dot{q}_{l}=\sum_{l} m_{k l} \dot{q}_{l}
$$

Aqui é importante perceber a sutileza: cada entrada da matriz de massa é constante e as velocidades generalizadas são, no máximo, quadráticas em cada coordenada, de modo que a derivação acima de fato resulta em algo linear nas velocidades generalizadas. 
Assim, usando todos os resultados anteriores, a "primeira parte das equações" de Lagrange em torno de um ponto de equilíbrio resulta, para cada $\mathrm{k}$, em:

$$
\frac{d}{d t}\left(\frac{\partial}{\partial \dot{q}_{k}}(T-V)\right)=\sum_{l} m_{k l} \ddot{q}_{l}
$$

Isto é, lembrando mais uma vez que $m_{k l}$ é apenas um número, essa primeira parte realmente é linear nas acelerações generalizadas. Resta agora a derivada de $\mathrm{T}$ e de $\mathrm{V}$ com relação às posições generalizadas, em torno do mesmo ponto de equilíbrio. A energia cinética dessa vez se anula, pois sendo a matriz de massa constante, $\mathrm{T}$ é função apenas das velocidades generalizadas:

$$
\frac{\partial T}{\partial q_{k}}=\frac{\partial}{\partial q_{k}}\left(\frac{1}{2} \dot{q}^{t} M \dot{q}\right)=0
$$

Resta investigar o que ocorre com o potencial. Intuitivamente, como iremos derivar uma vez em relação as posições, podemos imaginar que deveremos ter um potencial parabólico em torno do ponto de equilíbrio, afinal as equações de Lagrange o tornariam linear. Vamos proceder, então, nesse sentido: realizaremos um expansão em série de Taylor centrada no mesmo ponto de equilíbrio $\mathrm{P}_{0}$ da função potencial e pegaremos apenas os termos até segunda ordem. Assim:

$$
V\left(q_{1}, \ldots, q_{N}\right)=V_{0}+\sum_{k}\left(\frac{\partial V}{\partial q_{k}}\right)_{P_{0}} q_{k}+\frac{1}{2} \sum_{k, l}\left(\frac{\partial^{2} V}{\partial q_{k} \partial q_{l}}\right)_{P_{0}} q_{k} q_{l}+\ldots
$$

Sendo $\mathrm{P}_{0}$ um ponto de equilíbrio o segundo termo, de acordo com nosso critério de equilíbrio, se anula. Além disso, sendo $\mathrm{V}_{0}$ uma constante e que, portanto, some ao ser derivada podemos toma-la como sendo zero, o que nos dá:

$$
V=\frac{1}{2} \sum_{k, l}\left(\frac{\partial^{2} V}{\partial q_{k} \partial q_{l}}\right)_{P_{0}} q_{k} q_{l}=\frac{1}{2} \sum_{k, l} v_{k l} q_{k} q_{l}, \text { com } v_{k l}=\left(\frac{\partial^{2} V}{\partial q_{k} \partial q_{l}}\right)_{P_{0}}
$$

Como fizemos com a energia cinética, podemos interpretar $v_{k l}$ como sendo o elemento da linha $k$ e da coluna $l$ de uma matriz $\mathrm{NxN}$, que denotaremos por $\mathrm{K}$ e chamaremos de matriz de rigidez. 
Assim, definindo $q$ (sem subscrito) como sendo o vetor de posições generalizadas podemos reescrever a expressão anterior em notação matricial:

$$
V=\frac{1}{2} q^{t} K q, \operatorname{com} q^{t}=\left[\begin{array}{lll}
q_{1} & \ldots & q_{N}
\end{array}\right]
$$

Então a "segunda parte" das equações de Lagrange nos garantem para cada k:

$$
-\frac{\partial}{\partial q_{k}}(T-V)=\frac{\partial V}{\partial q_{k}}=\frac{\partial}{\partial q_{k}}\left(\frac{1}{2} q^{t} K q\right)=\sum_{l} v_{k l} q_{l}
$$

De novo, como cada $v_{k l}$ é apenas um número, o resultado final foi algo realmente linear em cada posição generalizada. Juntando os dois resultados obtidos, em notação matricial, as equações de movimento do sistema se tornam:

$$
M \ddot{q}+K q=0
$$

Veja que para cada coordenada $k$ (linhas dos vetores e das matrizes) a multiplicação entre matrizes e vetores nos retornam às somas nos índices $l$ (colunas das matrizes) que havíamos obtido anteriormente. Mas a questão principal é que finalmente chegamos em equações de movimento linearizadas em torno de um ponto de equilíbrio $\mathrm{P}_{0}$ e agora podemos analisar a estabilidade desse ponto de equilíbrio.

Para motivar o critério de estabilidade é válido realizar uma analogia com um conhecido sistema unidimensional, que aparece no estudo da dinâmica de um sistema em que uma massa $m$ se movimenta, sem atrito e devido à força de uma mola linear de constante $k$, após ser deslocada do equilíbrio:

$$
m \ddot{x}+k x=0
$$

Veja que a forma da equação é muito parecida com a que obtemos ao linearizar as equações de Lagrange. Em todo caso, nesse contexto do sistema massa-mola a constante $k$ só tem sentido físico se for positiva, o que garante soluções do tipo $x(t)=A e^{i \omega t}+B e^{-i \omega t}$ para a equação de movimento, isto é, soluções oscilatórias. Mas, matematicamente, nada impede que tenhamos uma equação análoga só que com $k$ negativo. Nesse caso, as soluções são do tipo $x(t)=A e^{\beta t}+B e^{-\beta t}$ e que, agora, indicam a possibilidade do sistema "explodir" para o infinito para $t$ grande (o que não acontece em um sistema que oscila). 
O importante dessa analogia é que a constante $k$ é que tem grande influência no comportamento do sistema massa-mola após pequenos deslocamentos em torno do equilíbrio: para valores positivos de $k$, obtemos soluções oscilatórias e, portanto, com certa estabilidade em torno do ponto de equilíbrio; enquanto que para $k$ negativo as soluções são exponenciais, podendo se afastar arbitrariamente do equilíbrio, o que caracteriza instabilidade.

Essa discussão toda é um indicativo que, ao menos intuitivamente, faz sentido que o equilíbrio do nosso sistema conservativo genérico N-dimensional tenha alguma relação com a matriz de rigidez K. Mas, se essa comparação não foi convincente o suficiente, vamos analisar o que exatamente significa essa matriz de rigidez. De maneira explícita, ela é dada por:

$$
K=\left[\begin{array}{ccccc}
\frac{\partial^{2} V}{\partial q_{1}^{2}} & \cdots & \frac{\partial^{2} V}{\partial q_{1} \partial q_{k}} & \cdots & \frac{\partial^{2} V}{\partial q_{1} \partial q_{N}} \\
\vdots & \ddots & & \vdots \\
\frac{\partial^{2} V}{\partial q_{k} \partial q_{1}} & & \frac{\partial^{2} V}{\partial q_{k}^{2}} & & \frac{\partial^{2} V}{\partial q_{k} \partial q_{N}} \\
\vdots & & & \ddots & \vdots \\
\frac{\partial^{2} V}{\partial q_{N} \partial q_{1}} & \cdots & \frac{\partial^{2} V}{\partial q_{N} \partial q_{k}} & \cdots & \frac{\partial^{2} V}{\partial q_{N}^{2}}
\end{array}\right]_{P_{0}}
$$

Ora, temos uma matriz $\mathrm{NxN}$ em que os elementos são as derivadas segundas de uma função avaliadas em um ponto (no caso a função potencial avaliada no ponto de equilíbrio), sendo que essa função é admitida diferenciável duas vezes e com derivadas continuas. Imediatamente segue disso que a matriz de rigidez nada mais é do que a chamada matriz hessiana da função potencial, afinal as duas são definidas de modo idêntico. Isso significa que essa matriz pode nos dar informações valiosas sobre o ponto de equilíbrio encontrado: é possível saber se $\mathrm{P}_{0}$ é ponto de máximo, mínimo ou sela da função potencial. E veja que isso de fato já nos é suficiente para estabelecermos um critério para a estabilidade do ponto de equilíbrio.

Pensando fisicamente, se $\mathrm{P}_{0}$ for um ponto de máximo do potencial, um pequeno deslocamento tende a diminuir a energia potencial, o que se traduz em energia cinética e, consequentemente, velocidade cada vez maiores e, portanto, um afastamento crescente desse ponto de equilíbrio. Se recuperarmos nossa definição de estabilidade de pontos de equilíbrio, esse comportamento se aproxima bastante do que se entende por equilíbrio instável: pequenas perturbações causando afastamento arbitrários. Além disso, como pontos de sela, por definição, se comportam como pontos de máximo ou mínimo, dependendo da direção em que há a perturbação, é natural incluirmos esses pontos como sendo de equilíbrio instável também. 
Por outro lado, se $\mathrm{P}_{0}$ é ponto de mínimo, um pequeno deslocamento significa um acréscimo na energia potencial. É de se esperar, então, que ao ser liberado nessa configuração, o sistema tenda a diminuir o potencial (afinal essa é a tendência natural de qualquer sistema), mas isso significa voltar justamente para o ponto de mínimo $\mathrm{P}_{0}$. Como temos uma situação em que pequenas perturbações retornam o sistema ao ponto original é possível classificar o equilíbrio como estável.

Tentando resumir todo o desenvolvimento dessa seção, seria interessante destacar o seguinte: ao analisarmos a estabilidade dos pontos de equilíbrio, estamos interessados no efeito que pequenas perturbações ao redor desses pontos causam no sistema; essa definição nos levou a ideia de linearização das equações de movimento em torno de um ponto de equilíbrio, o que fizemos obtendo energias cinética e potencial quadráticas para colocarmos nas equações de Lagrange; com as equações de movimento linearizadas, então, percebemos que a matriz de rigidez deveria exercer um papel fundamental na estabilidade de suas soluções; de fato, em seguida identificamos essa matriz como sendo a matriz hessiana da função potencial e, por fim, concluímos que o problema de classificação de estabilidade de pontos de equilíbrio se resumia a um problema de classificação de pontos críticos de uma função, associando estabilidade com pontos de mínimo do potencial e instabilidade com pontos de máximo ou de sela.

\section{APLICAÇÃO À ANÁliSE DO EMSC}

Com todas essas novas ferramentas em mãos, podemos voltar ao sistema proposto no EMSC e estudar a estabilidade dos pontos de equilíbrio. $\mathrm{Na}$ realidade, vamos nos limitar a analisar o equilíbrio em torno da origem $\mathrm{O}(0,0)$, uma vez que poderemos fazer conexões interessantes com a discussão sobre a bifurcação do sistema e o surgimento dos novos pontos de equilíbrio simétricos.

Para realizar essa análise, então, precisamos escrever as energias cinética e potencial quadráticas, que denotaremos por $T_{2}$ e $V_{2}$ respectivamente, em torno da origem. Começando pela energia cinética, é fácil ver que ela já é quadrática em $\dot{x}$ e $\dot{y}$, o que nos dá o seguinte:

$$
\left\{\begin{array}{c}
T=\frac{1}{2} m\left(\dot{x}^{2}+\dot{y}^{2}\right) \\
\dot{q}^{t}=\left[\begin{array}{ll}
\dot{x} & \dot{y}
\end{array}\right]
\end{array} \Rightarrow T_{2}=\left[\begin{array}{ll}
\dot{x} & \dot{y}
\end{array}\right]\left[\begin{array}{cc}
m & 0 \\
0 & m
\end{array}\right]\left[\begin{array}{c}
\dot{x} \\
\dot{y}
\end{array}\right]\right.
$$


Para o potencial quadrático precisaremos realizar todas as derivadas parciais de segunda ordem da função potencial que já obtivemos para montarmos a matriz de rigidez. Após um certo trabalho algébrico, é possível ver que:

$$
\left\{\begin{array}{l}
\left.\frac{\partial^{2} V}{\partial x^{2}}\right|_{(0,0)}=2 k(1-\alpha) \\
\left.\frac{\partial^{2} V}{\partial x \partial y}\right|_{(0,0)}=\left.\frac{\partial^{2} V}{\partial y \partial x}\right|_{(0,0)}=0 \Rightarrow V_{2}=\left[\begin{array}{ll}
x & y
\end{array}\right]\left[\begin{array}{cc}
2 k(1-\alpha) & 0 \\
0 & 2 k
\end{array}\right]\left[\begin{array}{l}
x \\
y
\end{array}\right] \\
\left.\frac{\partial^{2} V}{\partial y^{2}}\right|_{(0,0)}=2 k
\end{array}\right.
$$

Assim, colocando essas expressões nas equações de Lagrange, de fato obtemos um sistema linearizado em torno da origem:

$$
\left[\begin{array}{cc}
m & 0 \\
0 & m
\end{array}\right]\left[\begin{array}{l}
\ddot{x} \\
\ddot{y}
\end{array}\right]+\left[\begin{array}{cc}
2 k(1-\alpha) & 0 \\
0 & 2 k
\end{array}\right]\left[\begin{array}{l}
x \\
y
\end{array}\right]=0
$$

De qualquer forma, vamos nos voltar para a matriz (hessiana) de rigidez. Para analisarmos o equilíbrio, precisamos decidir se a origem é um ponto de máximo, mínimo ou sela e, para tanto, é preciso calcular seu determinante, que é dado por:

$$
\operatorname{det} K=4 k^{2}(1-\alpha) \Rightarrow\left\{\begin{array}{l}
\operatorname{det} K>0, \text { se } \alpha<1 \\
\operatorname{det} K=0, \text { se } \alpha=1 \\
\operatorname{det} K<0, \text { se } \alpha>1
\end{array}\right.
$$

Veja que o determinante depende do valor do nosso parâmetro $\alpha$, que, recordando, está relacionado com o estado original da mola. Consequentemente, a estabilidade do equilíbrio na origem também depende desse parâmetro, o que já indica de que forma faremos alguma relação com o diagrama de bifurcação. 
Vamos, então, analisar com maior cuidado as três situações possíveis para o determinante. Do cálculo, sabemos que para 2 dimensões um ponto é de mínimo se o seu determinante hessiano é positivo e o primeiro termo da diagonal principal também. Para $\alpha<1$ é justamente isso o que acontece, de modo que podemos concluir que na situação de molas inicialmente alongadas a origem é um ponto de equilíbrio estável.

Por outro lado, temos que quando o determinante é negativo o ponto em questão é um ponto de sela. Segue imediatamente que para $\alpha>1$ a origem é um ponto de sela do potencial e, portanto, quando as molas estão inicialmente comprimidas a origem apresenta um equilíbrio instável para o sistema. Por último, quando $\alpha=1$ o determinante é nulo e a análise via hessiano nos diz que nada se pode afirmar sobre o ponto em questão quanto sua classificação como extremante do potencial.

Nesse ponto, podemos retornar à bifurcação dos pontos de equilíbrio. Já mostramos que para $\alpha<1$ o único ponto de equilíbrio razoável era justamente a origem e que para $\alpha>1$ surgiam dois outros pontos simétricos em relação ao eixo y. Ora, $\alpha=1$ é justamente a situação limítrofe, em que o equilíbrio inicia sua bifurcação. Do ponto de vista da estabilidade desses pontos, a situação é bastante análoga: a origem começa como sendo um ponto de equilíbrio estável e, após a situação limítrofe, torna-se instável. É possível mostrar, ainda, que os novos pontos simétricos são, de fato, estáveis.

Para finalizar, é interessante deixar claro o que está acontecendo com o potencial conforme variamos $\alpha$. Para $\alpha<1$ temos que o potencial tem um único ponto de mínimo; esse ponto então se torna um ponto de sela para $\alpha>1$ o que origina dois "poços" de potencial, ou seja, dois novos mínimos simétricos em relação ao eixo $y$. Essa configuração no potencial incita a formação dos chamados atratores estranhos, fenômenos típicos de sistemas caóticos e presentes na teoria de caos desde seus "primeiros passos", mas cujos detalhes fogem do escopo desse texto. 


\section{SIMULAÇÃO}

Para a simulação, consideramos a massa da partícula $\mathrm{m}=50 \mathrm{~g}$, a distância entre as extremidades (pontos A e B) de 0,5m, e a constante elástica da mola $\mathrm{k}=10 \mathrm{~N} / \mathrm{m}$. Para cada sistema, há uma condição inicial diferente e um comprimento natural de mola diferente. É possível visualizar cada condição inicial nas imagens abaixo:
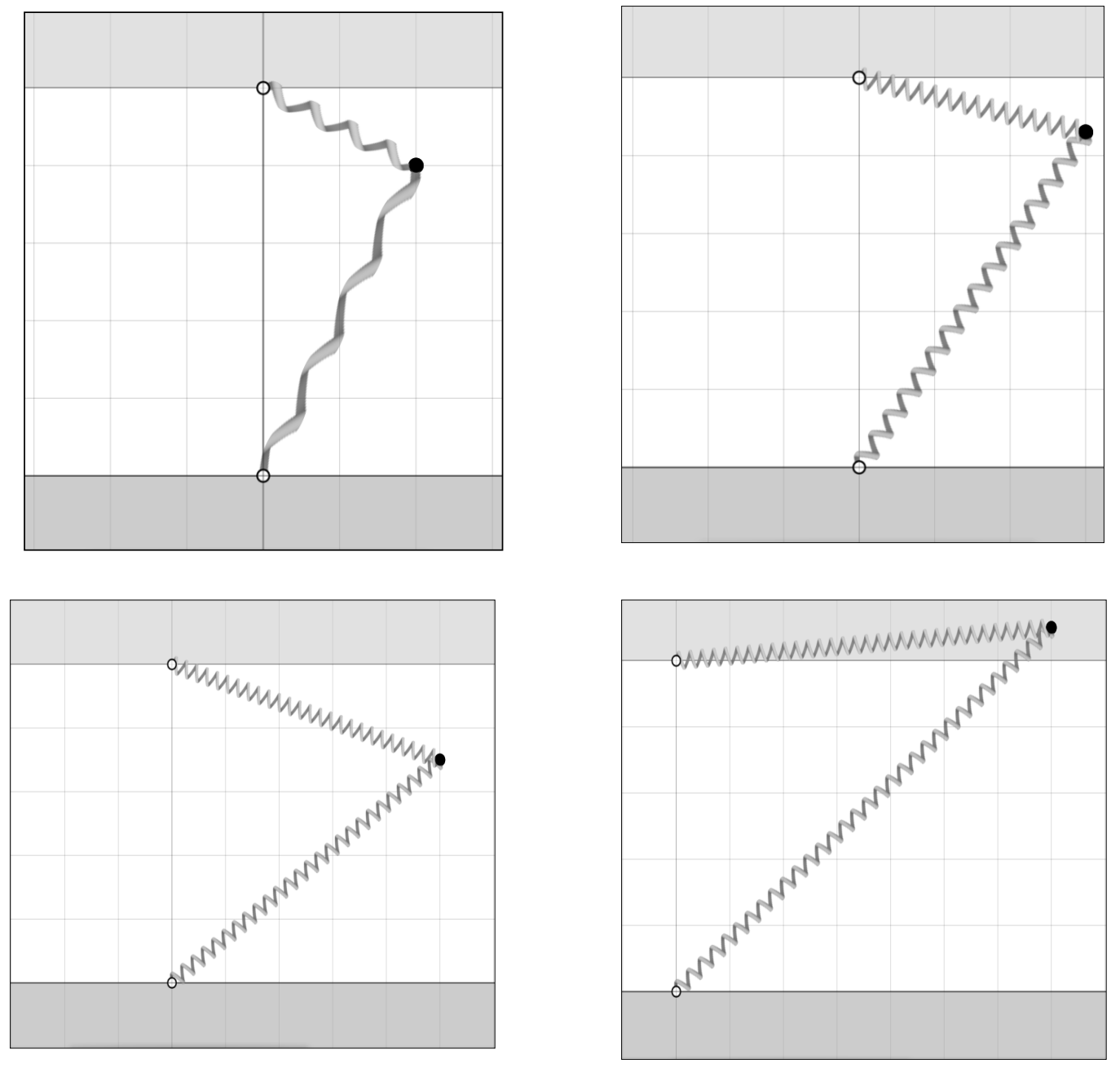

Analisaremos o sistema nestas quatro condições distintas, que consideramos interessantes para entender o comportamento do sistema.

1. A mola está inicialmente esticada $(\alpha=0.2)$ : Há um e somente um ponto de estabilidade na própria posição inicial da partícula.

2. A mola está em posição natural inicialmente $(\alpha=1)$ : Essa é uma condição limite do sistema. A partir deste valor, o sistema passa a ter 3 pontos de estabilidade, ao invés de somente 1. 
3. A mola está em compressão inicialmente $(\alpha=1.4)$ : O sistema admite dois pontos de equilíbrio estável simétricos e um ponto de equilíbrio instável na posição inicial.

4. A mola está numa compressão maior $(\alpha=2)$ : o comportamento do sistema é mais caótico e apresenta os três pontos de equilíbrio, como o anterior.

\subsection{POTENCIAL}

Como ferramenta de análise do sistema, obtemos o gráfico de energia potencial em função das coordenadas da massa. Os valores de energia potencial estão expressso em Joules, onde cores mais escuras correspondem a potenciais mais baixos e cores mais claras correspondem a potenciais maiores. Também é possível visualizar uma superfície $\mathrm{z}(\mathrm{x}, \mathrm{y})$, que representa um análogo ao potencial gravitacional.


Para as duas primeiras simulações é possível visualizar na sua superfície uma espécie de poço de potencial. Caso a partícula seja posicionada em qualquer ponto na vizinhança do ponto de estabilidade $(\mathrm{x}=0, \mathrm{y}=0)$, então a tendência é ela "cair" nesse posso, ou seja, oscilar em torno do ponto de estabilidade. No caso da segunda simulação é possível ver um certo alargamento do poço. Isso é devido ao valor de $\alpha=1$, um caso limite onde os "poços" tenderão a bifurcar para dois lados opostos. 

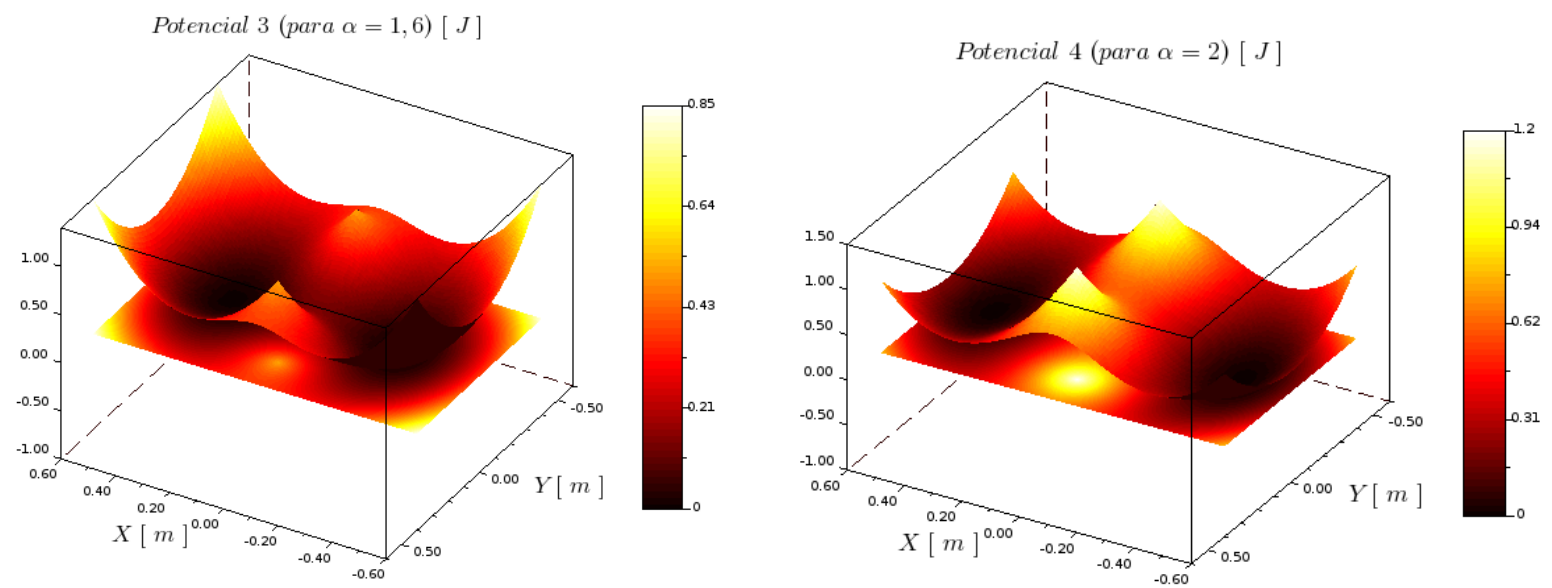

Para as duas últimas simulações, onde $\alpha>1$ já é possível ver dois poços distintos, e um ponto de sela na coordenada $(0,0)$. Este ponto de sela (que assemelha-se com uma sela de cavalo) é o que faz este ponto ser instável, pois se imaginarmos uma partícula na vizinhança com uma leve perturbação, ela tenderá a se afastar deste ponto. Por outro lado, na vizinhança dos poços, a partícula sempre tenderá a oscilar em torno de um poço (ou dos dois, como veremos a seguir). Os pontos mais claros desse potencial (onde se concentram dois "bicos") são as singularidades da força elástica, ou seja, o potencial não é diferenciável nesse ponto, logo não há gradiente que defina a direção da força nesse ponto. Enganosamente parece um ponto instabilidade no intervalo de análise, porém não é possível concluir nada a respeito da estabilidade nesse ponto por meio das ferramentas matemáticas.

Fisicamente, esses dois pontos representam a ligação das molas ao espaço. Logo, é de se esperar que elas não tenham nenhuma direção definida de força (é difícil imaginar para que direção uma mola comprimida a $l=0$ irá depois de ser liberada!)

\subsection{MOVIMENTO DA PARTÍCULA}

Agora que discutimos o suposto comportamento da partícula devido ao potencial presente, então foram feitas simulações dos movimentos nas quatro condições. A trajetória representa o movimento da partícula ao longo de um tempo de 10 segundos. A imagem mostra, além da trajetória da partícula, o potencial em escala de matiz (vermelho maior potencial, azul escuro menor potencial) e os dois pontos de fixação das molas (em preto). As partículas foram liberadas nas posições descritas pelas ilustrações anteriores. 


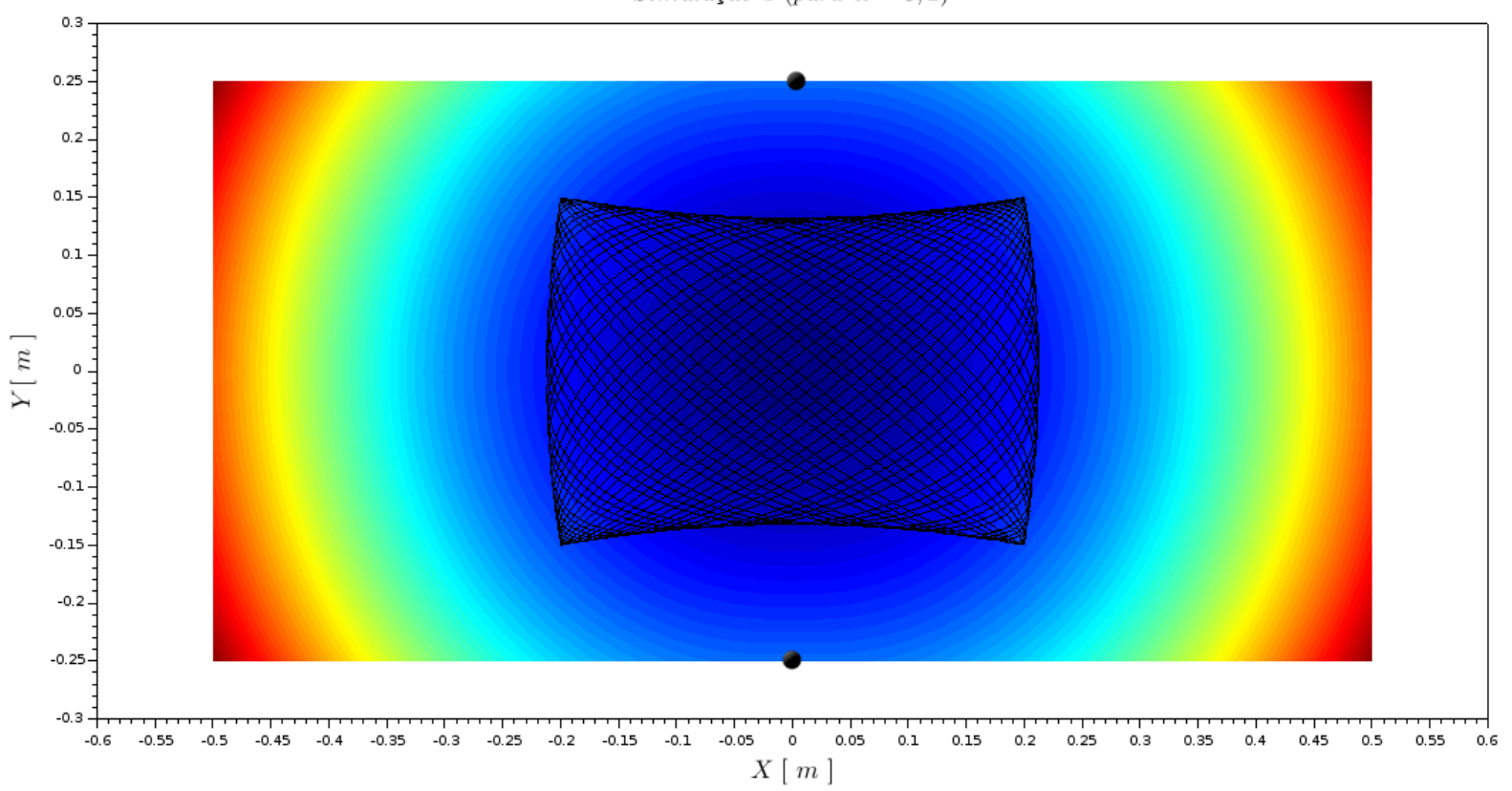

Simulação 2 (para $\alpha=1)$

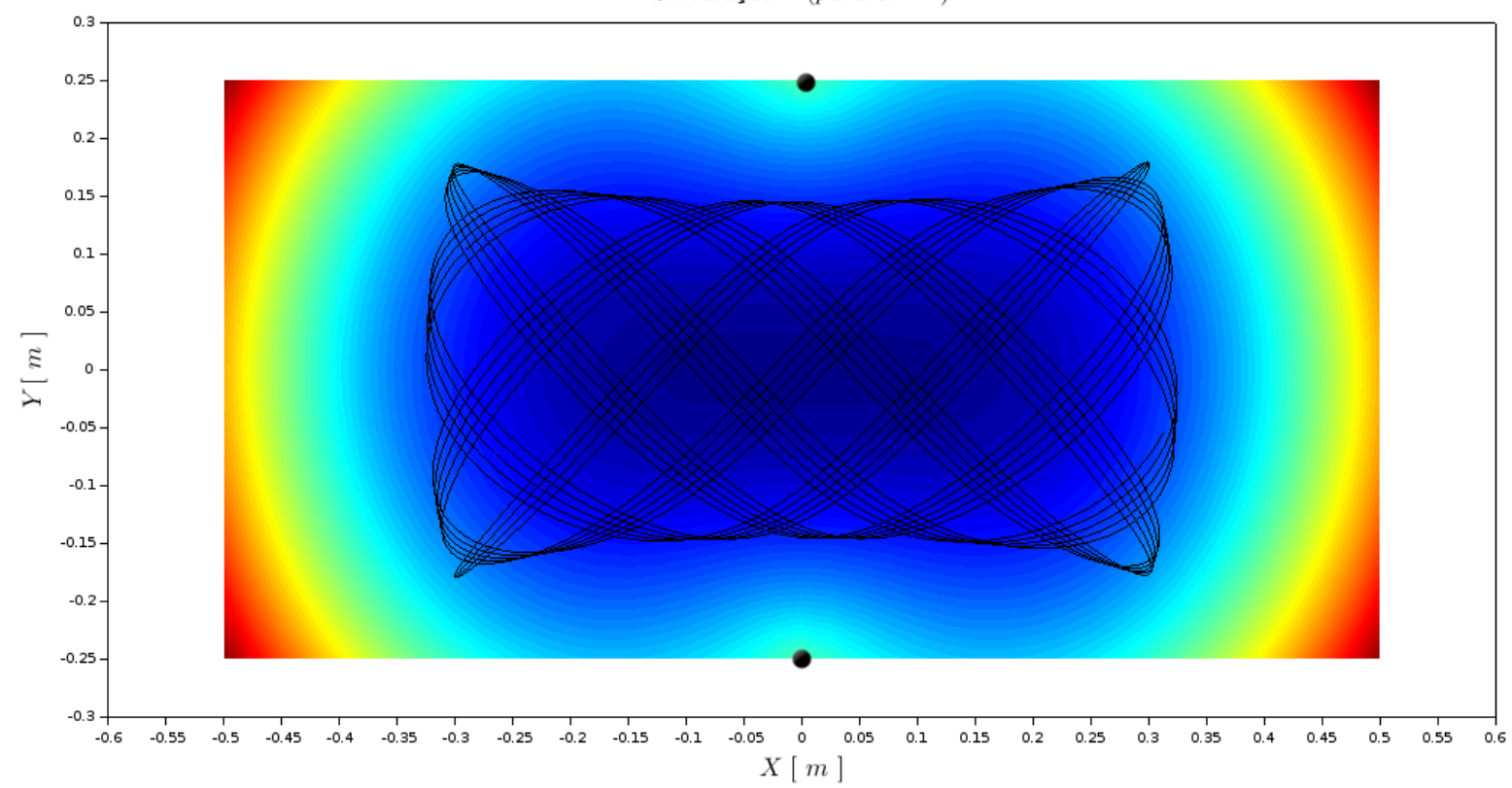

Como previmos, para as primeiras simulações a partícula oscila em torno do "poço" de potencial. É interessante notar que a partícula nos dois casos segue um movimento bem regular e periódico. Linearizando a equação da mola em torno de $(0,0)$ e resolvendo as equações diferenciais (que não faremos aqui) é possível notar uma parametrização de $(x, y)$ em função de t, em função de 
termos em seno e cosseno. Por conta disso, gera-se um padrão regular e simétrico que vimos nessa simulação. Existem curvas que exibem um comportamento similar que se chamam "curvas de Lissajous".


Para as duas últimas oscilações, devido a $\alpha>1$, há dois pontos de equilíbrio estáveis. Logo a partícula tende claramente a oscilar em torno deles. Como há energia o suficiente para a partícula "atravessar" de um lado para o outro, ela também tende a oscilar no outro poço. 
É interessante esse comportamento, pois uma leve diferença na posição inicial leva a trajetórias totalmente diferentes. Talvez a partícula oscile em torno de um poço, ou em torno dos dois. À primeira vista também não é possível ver se a partícula alternará de poço ou permanecerá no mesmo após uma oscilação, ou seja, para $\alpha>1$, o comportamento se torna caótico. A grosso modo, um sistema caótico é um sistema dinâmico que com pequenas perturbações iniciais, ele sofrerá grandes mudanças na sua trajetória, que a princípio não parecem tão óbvias de se prever.

\subsection{ESPAÇO DO FASES}

A fim de podermos visualizar comportamentos interessantes sobre o sistema, às vezes é útil termos um espaço de fases. Um espaço de fases é um espaço n-dimensional constituído por $\mathrm{n}$ variáveis (arbitrárias e que nos interessam) de um sistema dinâmico. No caso, podemos considerar um espaço de fases constituído por $\mathrm{x}$ versus velocidade em $\mathrm{x}$ ou y versus velocidade em $\mathrm{y}$.

Os espaços de fase que escolhemos estão relacionados com energia potencial e energia cinética. Plotando o gráfico deles em cada condição podemos obter resultados interessantes.
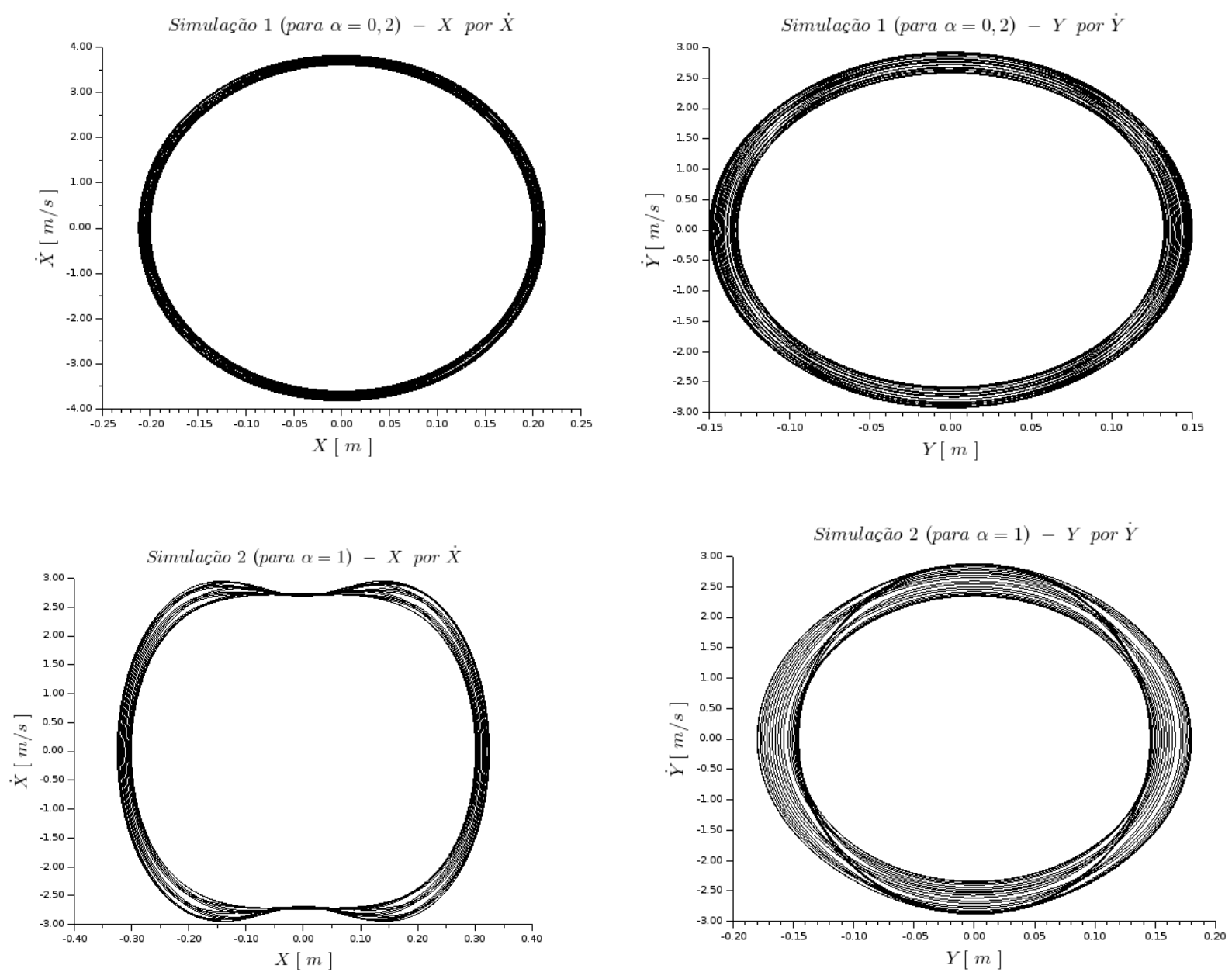
Para a primeira simulação, podemos ver que o espaço de fases nos dois gráficos são um conjunto de curvas fechadas que se aproximam para uma elipse. Isso é um comportamento característico para sistemas que apresentam estabilidade. Também podemos interpretar a curva como sendo aproximadamente uma elipse que satisfaz a equação:

$$
\frac{x^{2}}{a^{2}}+\frac{\dot{x}^{2}}{b^{2}}=1
$$

Sendo a e b constantes quaisquer. Mas para pequenas perturbações em x não considerando o movimento em y (como sendo desprezível), então o potencial $\mathrm{V}$ da mola e a energia cinética $\mathrm{K}$ (escolhendo-se as constantes adequadas) podem ser aproximados como:

$$
K \approx \frac{\dot{x}^{2}}{b^{2}} e V \approx \frac{x^{2}}{a^{2}} \Rightarrow K+V=1
$$

A última equação impõe que a energia mecânica $(\mathrm{V}+\mathrm{K})$ é constante, o que condiz com a situação dos sistemas conservativos que estamos analisando. Claro que é apenas uma interpretação aproximada e sujeita a imprecisões, mas é possível ter um pouco de intuição sobre o porquê das curvas fechadas, neste caso em que as oscilações são regulares, relativamente pequenas e o potencial é quase simetricamente radial.
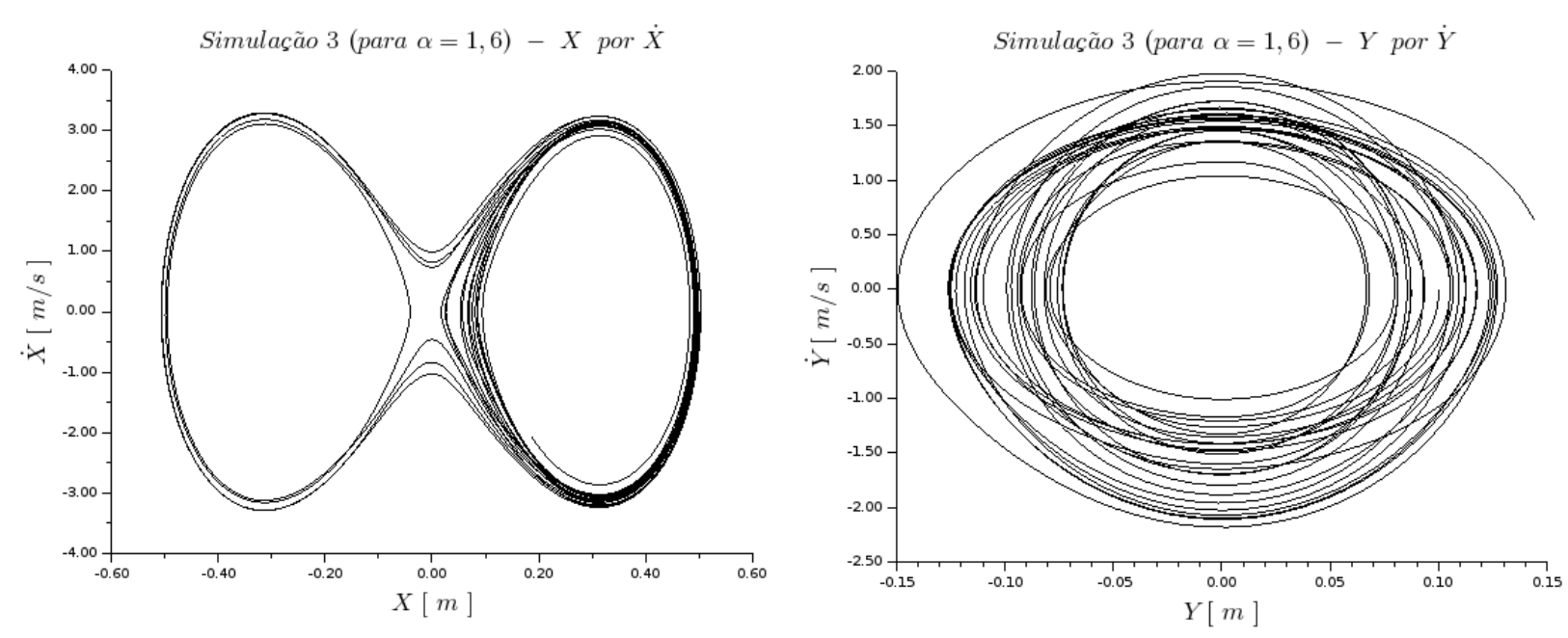

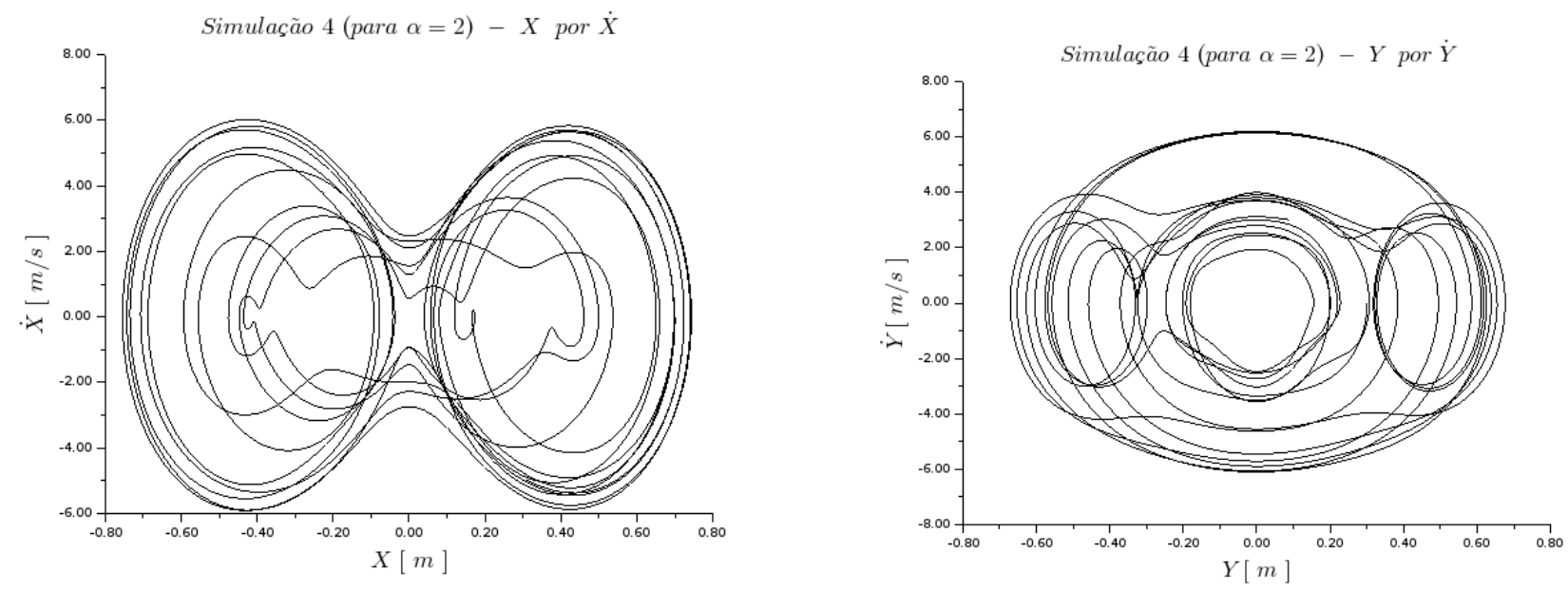

Para estas duas últimas simulações é possível visualizar padrões interessantes. O que mais se destaca é o fato de ter duas "órbitas" que aparentemente representam os dois pontos de estabilidade do sistema. Para a última simulação, mais caótica, essas curvas se comportam muito menos regularmente e seguem uma órbita muito menos definida. Intuitivamente o conceito desses espaços de fases apresentam um tipo de comportamento de periodicidade e estabilidade do sistema dinâmico. 